\title{
Pioneers 10 and 11 Deep Space Missions
}

\section{Palmer Dyal}

February 1990

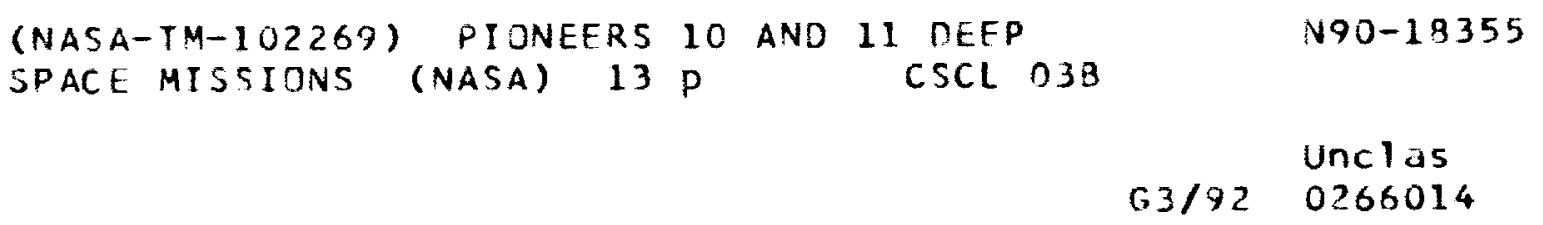

\section{N/SA}

National Aeronautics and

Space Administration 


\section{-}

$\ldots$

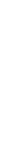


NASA Technical Memorandum 102269

\section{Pioneers 10 and 11 Deep Space Missions}

Palmer Dyal, Ames Research Center, Moffett Field, California

February 1990

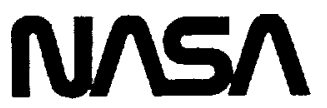

National Aeronautics and

Space Administration

Ames Research Center

Moffett Field, California 94035 

PIONEERS 10 AND 11 DEEP SPACE MISSIONS

P. Dyal

Space Research Directorate, NASA Ames Research Center, Moffett Field, California 94035

,

\section{ABSTRACT}

Pioneers 10 and 11 were launched from Earth March 2, 1972, and April 5, 1973, respectively. They were the first spacecraft to explore the asteroid belt and the first to encounter the giant planets, Jupiter and Saturn. The Pioneer 10 spacecraft is now the most distant man-made object in our solar system and is farther from the Sun than all nine planets. It is $47 \mathrm{AU}$ from the Sun and is moving in a direction opposite to that of the Sun's motion through the galaxy. Pioneer 11 is $28 \mathrm{AU}$ from the Sun and is raveling in the direction opposite of Pioneer 10, in the same direction as the Sun moves in our galaxy. These two Pioneer spacecraft have provided the first large-scale, in situ measurements of the gas and dust surrounding a star, our Sun. Since launch, the Pioneers have measured large-scale properties of the heliosphere during more than one complete 11-year solar sunspot cycle, and have measured the properties of the expanding solar atmosphere, the transport of cosmic rays into our heliosphere, and the high-energy trapped radiation belts and magnetic fields associated with the planets Jupiter and Satum. Accurate Doppler tracking of these spin-stabilized spacecraft has been used to search for differential gravitational forces from a possible trans-Neptunian planet and to search for gravitational radiation. Furure objectives of the Pioneer 10 and 11 missions are to continue measuring the large-scale properties of our heliosphere and to search for its boundary with interstellar space.

\section{GENERAL MISSION DESCRIPTION}

The Pioneer 10 spacecraft launched March 2, 1972, is the most distant man-made object in our solar system. Mission studies initiated in the early 1960 s came to fruition in 1969 when Ames Research Center was assigned the exploratory Pioneer Jupiter Project. Three spacecraft were built to achieve the mission objective; the first, for lesting, which is now in the National Air and Space Museum, the second, named Pioneer 10, and the third launched April 5. 1973, named Pioneer 11. These two spacecraft were the first to be launched with enough energy to escape the solar system. After exploring the regions of space beyond the orbit of Mars, both spacecraft encountered Jupiter and then Pioneer 11 was targeted for Satum. Pioneer 11 was launched one year after Pioneer 10 so its trajectory could be modified depending on the condition of Pioneer 10 after it passed through the asteroid belt and Jupiter's radiation belts.

The missions are expected to last three decades and the following objectives have evolved:

(1) Explore the interplanetary medium beyond the orbit of Mars.

(2) Investigate the nature of the asteroid belt and assess its hazard to future missions.

(3) Explore the environment of Jupiter.

(4) Explore the environment of Saturn.

(5) Explore the outer heliosphere and potentially pass into interstellar space.

Many individuals and institutions were required to implement these two missions. The Pioneer 10 and 11 Project was managed by Ames Research Center and NASA Headquarters, the Atlas Centaur booster by Lewis Research Center, launch operations by Kennedy Space Center, the Deep Space Network tracking by Jet Propulsion Laboratory (JPL), the radioisotope thermoelectric power generators by the Atomic Energy Commission, and the spacecraft was built by TRW Systems Group. Six universities and four NASA centers provided the scientific experiments for the project; they are listed in Table 1 . 
TABLE 1 Experiments and Principal Investigators, Pioneer 10 and 11

\begin{tabular}{ll}
\hline Helium Vector Magnetometer & $\begin{array}{l}\text { Dr. Edward J. Smith } \\
\text { Jet Propulsion Laboratory }\end{array}$ \\
Plasma Analyzer & $\begin{array}{l}\text { Dr. Aaron Barnes } \\
\text { Ames Research Center }\end{array}$ \\
Charged Particle Instrument & $\begin{array}{l}\text { Dr. John A. Simpson } \\
\text { University of Chicago }\end{array}$ \\
Cosmic Ray Telescope & $\begin{array}{l}\text { Dr. Frank B. McDonald } \\
\text { Goddard Space Flight Center }\end{array}$ \\
Geiger Tube Telescope & $\begin{array}{l}\text { Dr. James A. Van Allen } \\
\text { University of Iowa }\end{array}$ \\
Trapped Radiation Detector & $\begin{array}{l}\text { Dr. R. Walker Filluis } \\
\text { University of California, San Diego }\end{array}$ \\
Ultraviolet Photometer & $\begin{array}{l}\text { Dr. Dartell L. Judge } \\
\text { University of Southern California }\end{array}$ \\
& Dr. John D. Anderson \\
Celestial Mechanics & Jet Propulsion Laboratory \\
& Dr. Tom Gehrels \\
Unaging Photopolarimetry &
\end{tabular}

The two spacecraft and most of the scientific instruments are in excellent condition. Data are being acquired on a daily basis at 16 bits per second with the 70-m diameter antennas of the JPL Deep Space Network. This telemetry command and receiving capability should permit the spacecraft and experiments to be operated to the end of the century. In August 1989, Pioneer 10 was 47.16 AU from the Sun and the round-trip light time was 13 hr 6 min. Pioneer 11 was $28.99 \mathrm{AU}$ from the Sun and the corresponding command and receive time was $7 \mathrm{hr} 58 \mathrm{~min}$.

Figure 1 shows the prominent physical features of the spacecraft. It is a highly reliable spacecraft of relatively simple design in which many of the components and subsystems have successfully performed on earlier missions. It has a thermally controlled equipment compartment with two sections, one is hexagonally shaped and contains electronic units and the propellant tank, and the other is a bay which contains most of the scientific sensors and their associated electronics (the magnetometer sensor and two meteoroid detectors are external). Forward of the equipment comparment is a $2.7-\mathrm{m}$ diameter parabolic reflector for the high-gain antenna. Mounted on a tripod structure forward of the reflector are the medium-gain antenna and the feed for the high-gain antenna. Three appendages are stowed within a $2.7-\mathrm{m}$ cylindrical envelope at launch; they are shown in their deployed positions attained within an hour after launch. Two pairs of radioisotope thermoelectric generators (RTGs) are extended approximately $1.8 \mathrm{~m}$ at $120^{\circ}$ spacing. The RTGs are retained in a stowed position for launch next to the equipment compartment and under the antenna reflector. The magnetometer sensor is located on the end of a long folding boom which, in the deployed condition, extends $5.2 \mathrm{~m}$ radially from the instrument side of the equipment comparment.

Six 4-N hydrazine thrusters are located in three clusters near the perimeter of the 2.7-m reflector. Two pairs of thrusters are aligned parallel to the spin axis for precession and velocity correction maneuvers; two thrust tangentially for spin control. Other external features include a mast-mounted, omnidirectional antenna directed aft, and a Sun sensor mounted near one of the thruster assemblies which determines the spacecraft's position in the spin cycle. Two large light shields are associated with the stellar-reference assembly, and with an optical asteroid/meteroid detector.

The spacecraft electronic assemblies are located in the central hexagonal portion of the compartment, surrounding a 42-cm diameter hydraxine tank. Most of the scientific instruments' electronic units and internally mounted sensors are in an instrument bay mounted on one side of the central hexagon. This equipment compartment is contained within a structure of aluminum honeycomb which provides support and meteroid protection. It is covered with insulation which, together with louvers under the mounting platform, provide thermal control. Adequate temperature is provided by dissipation of the 90 watts of electrical power within the compartment and by use of radioisotope and electrical heaters. 


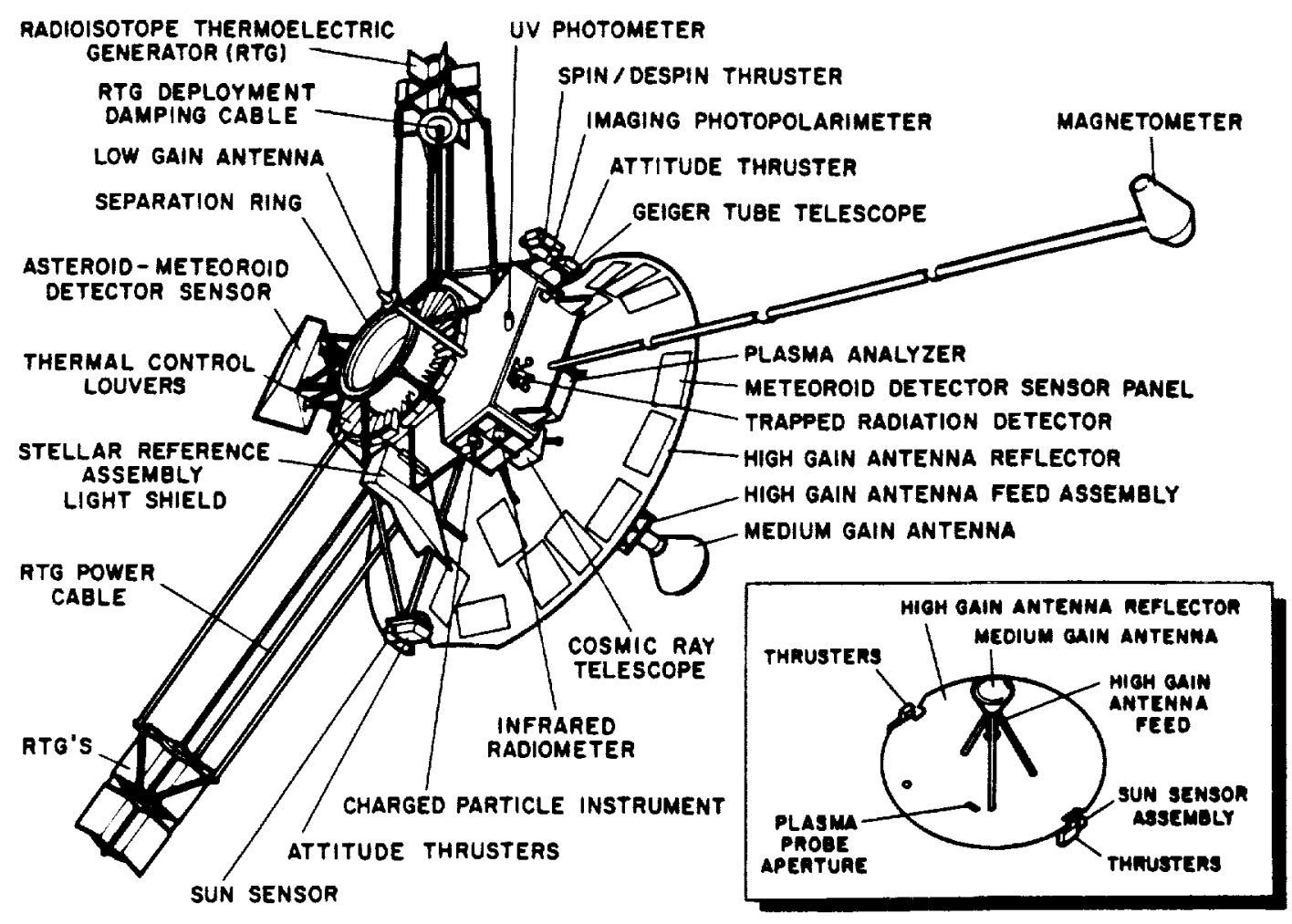

Fig. 1. Pioneer/Jupiter spacecraft.

The spacecraft is attitude-stabilized by spinning about an axis which is parallel to the axis of the $2.7-\mathrm{m}$ reflector, the nominal spin rate for Pioneer 10 is $4.8 \mathrm{rpm}$. Pioneer 11 spins at approximately $7.8 \mathrm{rpm}$ because of an inoperative thruster. During the mission, an Earth-pointing artitude is required to illuminate the Earth with the narrow-beam, high-gain antenna. Periodic attitude adjustments are required throughout the mission to compensate for the variation in the heliocentric longitude of the Earth-spacectaft line. In addition, correction of launch vehicle injection errors were required to provide the desired Jupiter encounter trajectory and Saturn (Pioneer 11 only) encounter trajectory. These velocity vector adjustments involve reorienting the spacecraft to direct the thrust in the desired direction.

Thrusters are used to control the spin of the spacecraft, its attitude, and its velocity, as shown in Figure 1. The precession maneuvers can be open-loop, for orientations toward or away from Earth-pointing, or closed-loop, for homing on the uplink radio frequency transmission from Earth.

For attitude control, a star sensor (Canopus) and two sunlight sensors provide reference, and three pairs of thrusters located near the rim of the antenna dish provide for orientation and roll maneuver. The star sensor on Pioneer 10 has been inoperative since the Jupiter encounter. About $44.7 \mathrm{lb}$ of hydrazine propellant remain on Pioneer 10 and about $11.3 \mathrm{lb}$ remain on Pioneer 11 . The magnetometer boom incorporates a hinged viscous-dampening mechanism at its attachment point, for passive nutation control. The remaining hydrazine propellant on Pioneer 10 and 11 is adequate to support the attitude control maneuvers well into the next century and will not limit the mission lifetime.

A unique characteristic of this spacectaft is the use of RTGs as the primary source of electrical power, making it independent of the Sun. Each of the four space-proven SNAP-19 RTGs converts 5 to 6 percent of the heat released from plutonium dioxide radioactive decay to electrical power. The RTG power is greatest at 4.2 volts and an inverter boosts this to 28 volts for distribution. The RTG lifetime is degraded at lower currents, therefore, voltage is regulated by shunt dissipation of excess power. At launch, the four RTGs supplied about 160 watts on each spacecraft as shown in Figures 2 and 3. Examination of all subsystems on both spacecraft to date indicates that RTG power is the most critical in determining mission lifetime. Approximately 80 watts minimum are required to operate the spacecraft and a small subset of the scientific instruments. As seen in Figures 2 and 3, the RTGs decay at different rates. It is projected that Pioneer 11 will last until 1994 and Pioneer 10 until 1997 before the power drops to 80 watts and the shunt current decreases to zero. Presently the scientific instruments on Pioneer 11 are sequentially tumed on and off to time-share the remaining power. It is expected that Pioneer 10 will be required to start a power-sharing program during September 1989. 


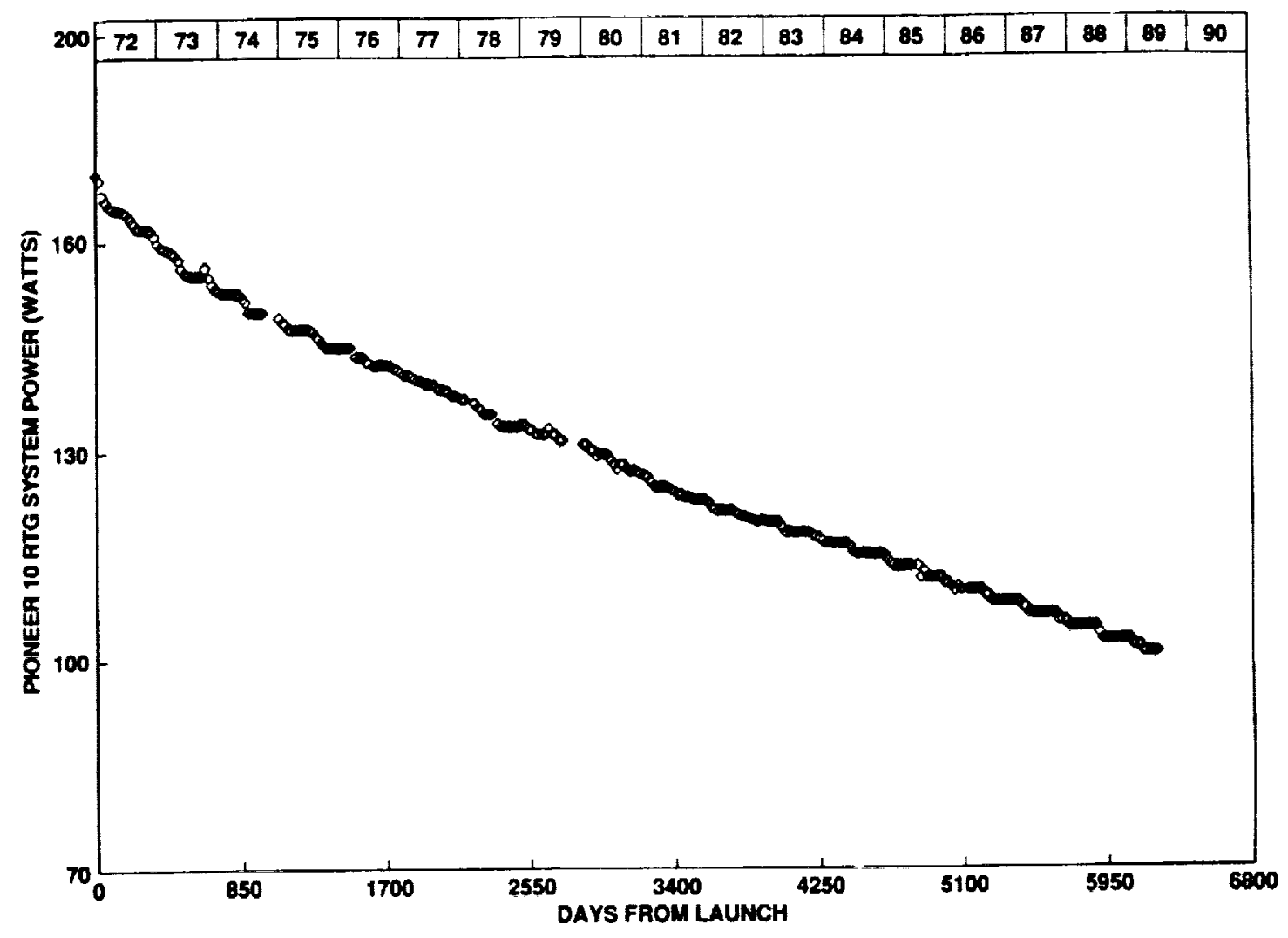

Fig. 2. Pioneer 10 RTG system power history.

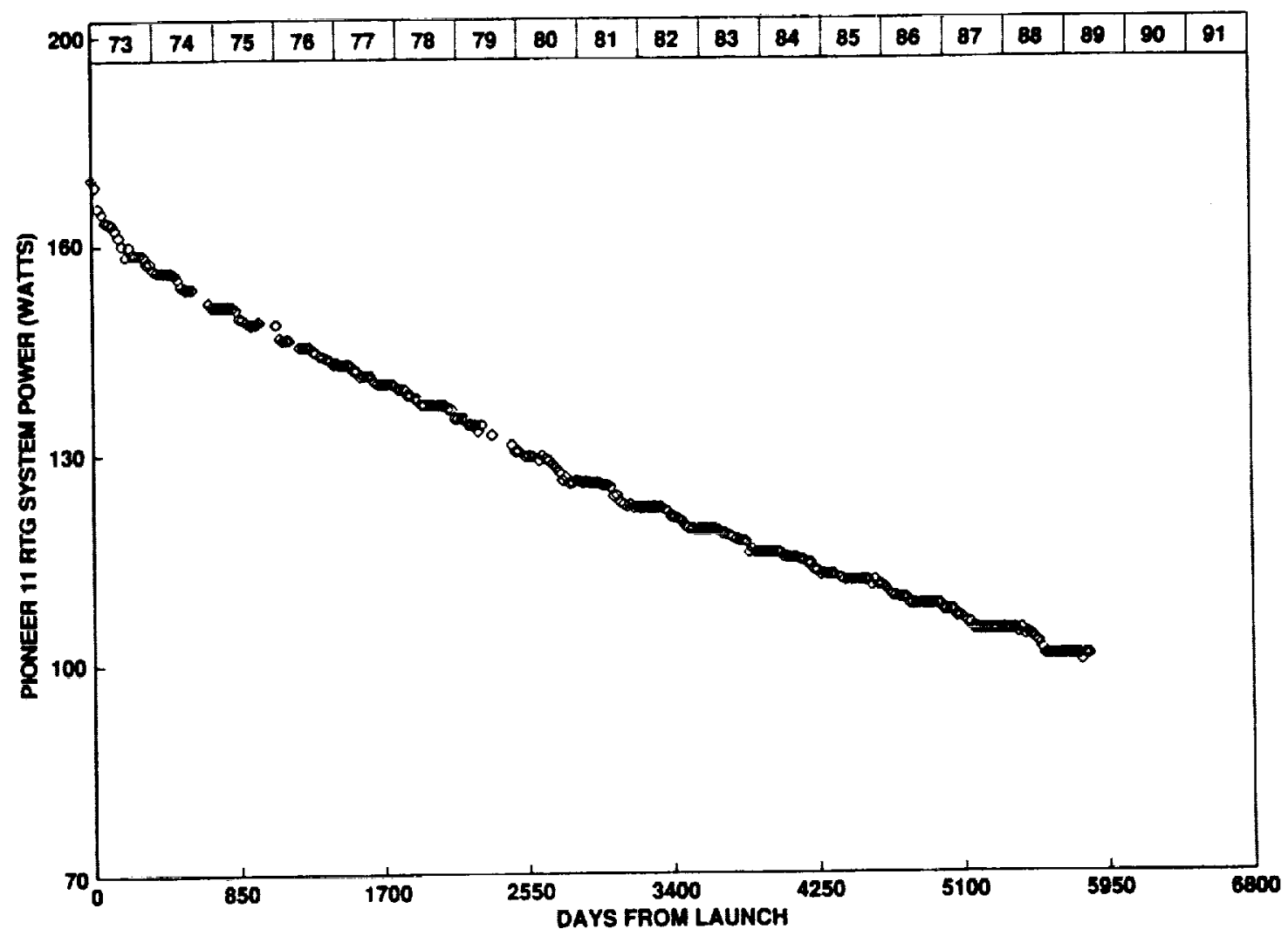

Fig. 3. Pioneer 11 RTG system power history. 
The launch vehicle for Pioneer 10 and 11 was the three-stage Atlas/Centaur/TE-364-4, shown in Figure 4 with dimensions in feet and inches. The long-tank Atlas is a space booster version of the Series $\bar{D}$ intercontinental ballistic missile, and when used within the second-stage Centaur vehicle is designated the "Atlas SLV-3C." The Centaur is an operational high-energy space vehicle which uses liquid hydrogen for fuel. The Atlas/Centaur System required the TE-364-4 third stage for Jupiter injection velocities. This launch vehicle boosted each spacecraft to begin the flight to Jupiter at about $51,680 \mathrm{~km} / \mathrm{hr}$.

Pioneers 10 and 11 are proceeding in approximately opposite directions, the latter toward the nose of the heliosphere, and the former down the hypothetical tail of the heliosphere, as shown in Figure 5. Both Voyager spacecraft are proceeding in the same quadrant of the solar system as Pioneer 11. These four spacecraft form a unique set of measurement probes to measure the three-dimensional, large-scale structure of the Sun's corona. Pioneers 10 and 11 have asymptotic escape velocities from the solar system of 2.4 and 2.2 AU/year, respectively, and there is a reasonable expectation that Pioneer 10 will penetrate the heliosphere boundary and pass into the interstellar medium before the radioisotope power supply outputs decrease below the level to sustain spacecraft requirements.

Currently nine scientific experiments are being conducted on the two nearly identical spacecraft. A list of the principal investigators and their experiments is shown in Table 2 and an abbreviated list of the instrument properties is given in Table 3. These instruments are all operating normally except for the helium vector magnetometer on Pioneer 10 which failed one year after the Jupiter encounter and the imaging photopolarimeter which is only used for aspect determination on Pioneer 10. Two meteoroid detection experiments and a Jovian Infrared Thermal Structural Experiment were also tumed off after their primary objectives were completed. References $/ 1 /$ and $/ 2 /$ give a more detailed description of the experiments and their operation during the early phases of the two missions.
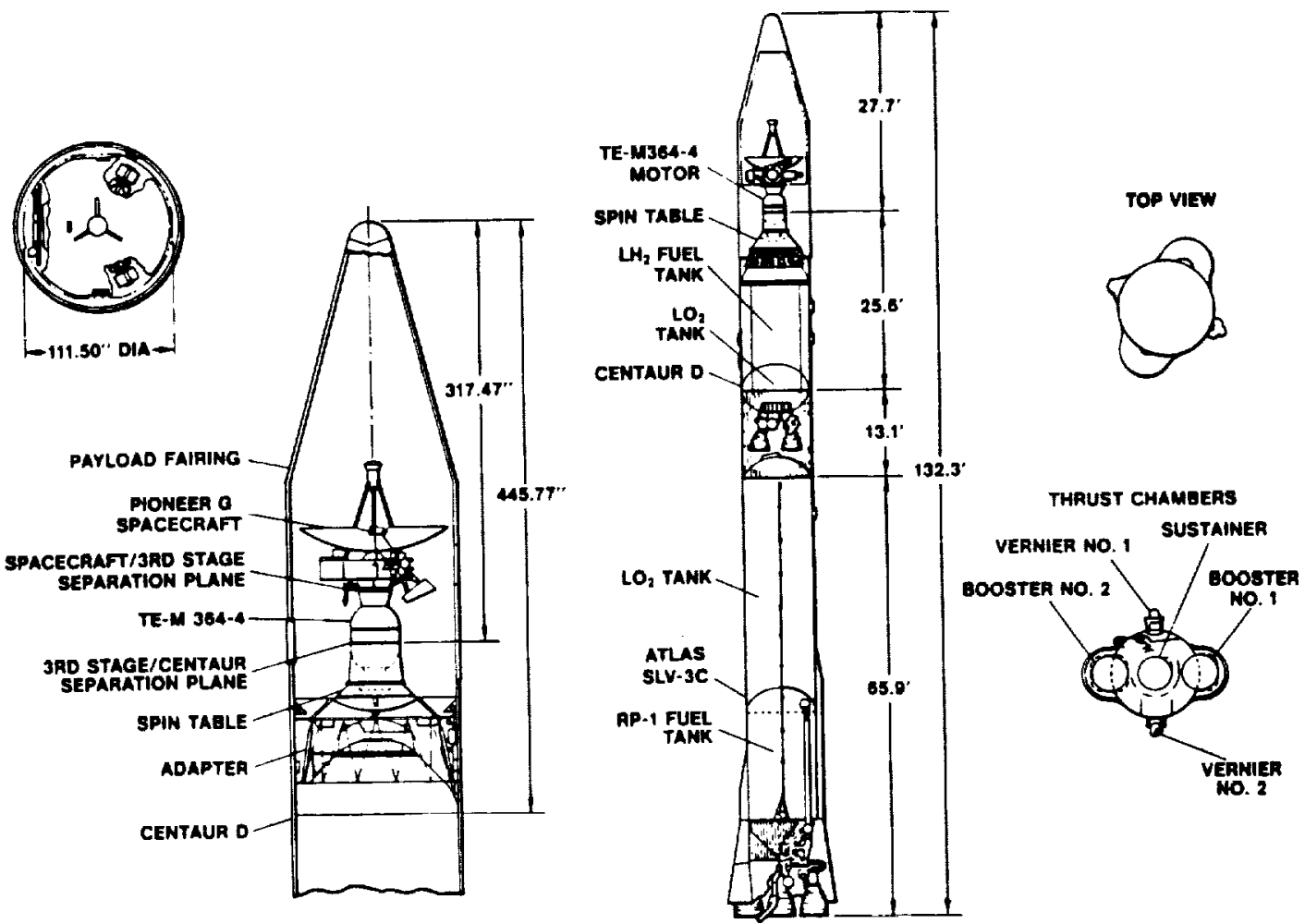

Fig. 4. Pioneer 10/11 launch vehicle. 


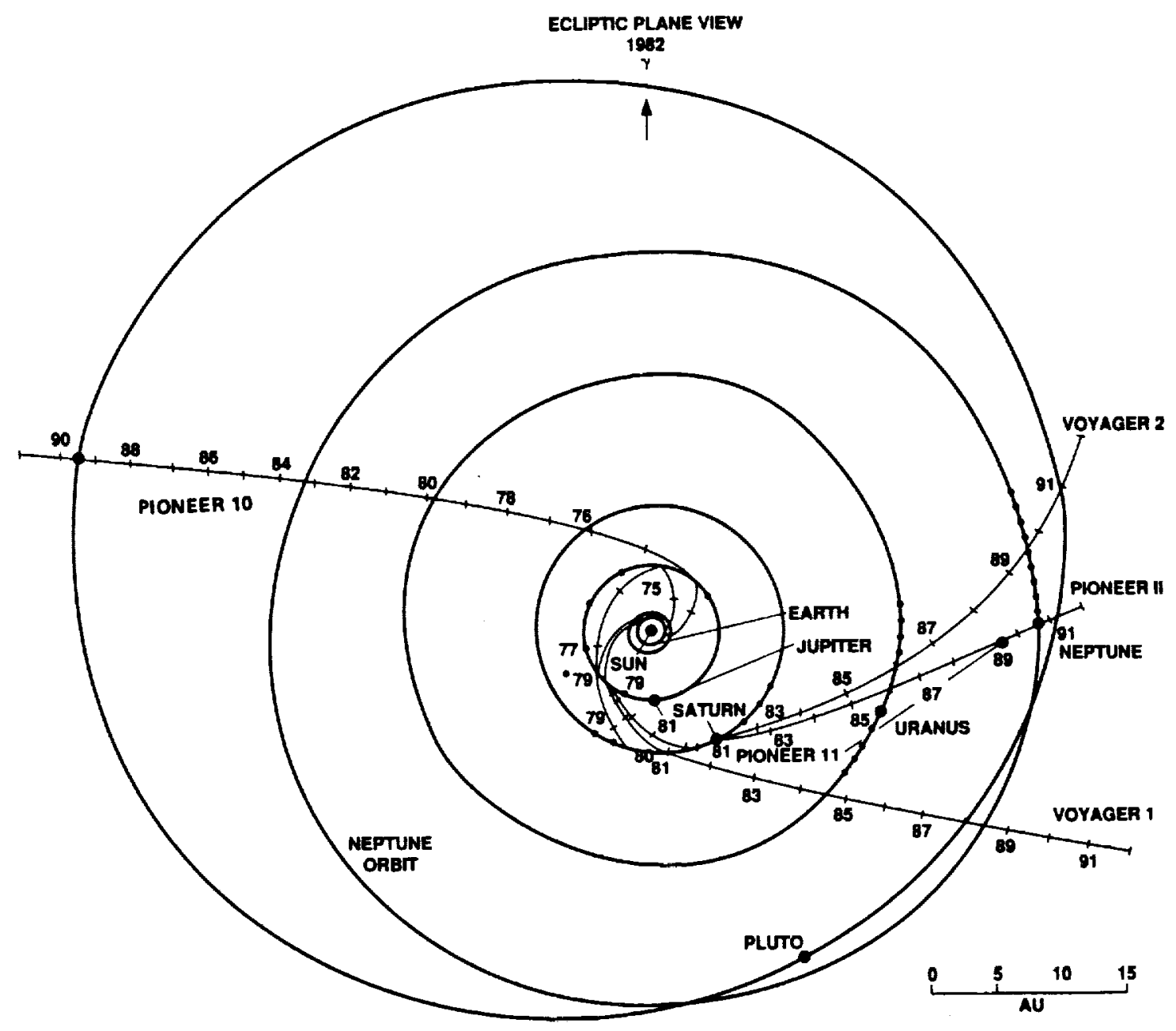

Fig. 5. Trajectories of Pioneer 10 and 11, and Voyager 1 and 2. 
TABLE2 Current Scientific Experiment Properties

\begin{tabular}{|c|c|c|}
\hline Experiment & Detection Principle & Sensitivity \\
\hline 1. Helium Vector Magnetometer & Helium vapor, Zeeman effect & $\begin{array}{l}0.01 \text { gamma; range } \pm 4.0 \text { gamma to } \\
\pm 1.41 \text { gauss }\end{array}$ \\
\hline 2. Plasma Analyzer & Electrostatic analyzer, channeltrons & $\begin{array}{l}0.1 \text { to } 18 \mathrm{KeV} \text { protons, } 0.001 \text { to } \\
0.5 \mathrm{KeV} \text { electrons; } 10^{2} \text { to } \\
10^{9} \mathrm{P}^{+} \mathrm{CM}^{-2} \mathrm{SEC}^{-1}\end{array}$ \\
\hline 3. Charged Particle Instument & $\begin{array}{l}\text { Two Si detector multi-element tele- } \\
\text { scopes electron detector and fission } \\
\text { cell }\end{array}$ & $\begin{array}{l}\text { Discriminates protons through oxy- } \\
\text { gen; } 0.5 \text { to } 500 \mathrm{MeV} / \text { nucleon; elec- } \\
\text { trons } 3 \text { to } 30 \mathrm{MeV} \text {; integral fluxes for } \\
\mathrm{P}^{+} \text {through } \mathrm{Fe}^{+}>500 \mathrm{MeV} / \text { nucleon }\end{array}$ \\
\hline 4. Cosmic Ray Telescope & Three Si solid state telescopes & $\begin{array}{l}\text { I } \mathrm{P}^{+} \text {to } \mathrm{Ne} ; 3 \text { to } 22 \mathrm{MeV} / \text { nucleon } \\
\text { II. electrons } 0.5 \text { to } 1.0 \mathrm{MeV} ; \mathrm{P}^{+} 0.05 \\
\text { to } 20 \mathrm{MeV} \\
\text { III. } \mathrm{P}^{+} \text {and } \mathrm{He} ; 22 \text { to } 800 \mathrm{MeV} / \\
\text { nucleon }\end{array}$ \\
\hline 5. Geiger Tube Telescope & $\begin{array}{l}\text { Geiger-Mueller tubes, } 7 \text { each } \mathrm{Si} \\
\text { solid-state detector }\end{array}$ & $\begin{array}{l}\mathrm{P}^{+}>70 \mathrm{MeV} ; 0.2 \text { to } 10^{6} \text { counts } / \mathrm{sec} ; \\
\tau>0.06 \mathrm{MeV} ; \mathrm{P}+0.6 \text { to } 3.4 \mathrm{MeV}\end{array}$ \\
\hline 6. Trapped Radiation Detector & Cerenkov detector: alcohol & $\begin{array}{l}\text { - } 0.5 \text { to } 12 \mathrm{MeV} ; \mathrm{P}^{+}>80 \mathrm{MeV} ; \mathrm{Z}^{+} \\
>500 \mathrm{MeV} / \text { nucleon }\end{array}$ \\
\hline 7. Ultraviolet Photometer & $\begin{array}{l}\text { Al filter with channeltron for hydro- } \\
\text { gen Lyman alpha and LiF target cath- } \\
\text { ode for } 584 A \text { helium }\end{array}$ & $\begin{array}{l}200 \text { to } 1400 A \text {; field of view } 1.15^{\circ} \times \\
9.3^{\circ} \mathrm{H} \text { and } \mathrm{He} \text { lines at } 1216 \AA \text { and } \\
584 \AA\end{array}$ \\
\hline 8. Celestial Mechanics & $\begin{array}{l}\text { Doppler tracking of spacecraft at } \\
2110 \mathrm{MHZ} \text { uplink and } 2292 \mathrm{MHZ} \\
\text { downlink }\end{array}$ & Acceleration $>1 \mathrm{~mm} / \mathrm{sec}^{2}$ \\
\hline 9. Imaging Photopolarimeter & $\begin{array}{l}2.54 \mathrm{~cm} \text { Maksutov telescope } \\
\text { Wollaston prism }\end{array}$ & $\begin{array}{l}\text { Field of view } 0.028^{\circ} \text { square, red and } \\
\text { blue filters. } 64 \text { shades of gray. Used } \\
\text { spacecraft spin for scanning. }\end{array}$ \\
\hline
\end{tabular}

\section{SYNOPSIS OF SCIENTIFIC RESULTS}

Pioneer 10 entered the center of the asteroid belt in September 1972 and the meteoroid detectors indicated much less material at small particle sizes than had been anticipated. This spacecraft then made its closest approach to Jupiter on December 3,1973 , at $130,354 \mathrm{~km}$ above the cloud tops and then proceeded out to deep space in a direction opposite to that of the Sun's motion in the galaxy. Pioncer 11 followed about one year later and passed through the asteroid belt by March 1974. Its meteoroid experiments showed a decline in small-sized particles $(0.001$ $\mathrm{mm}$ ) in the belth an even distribution of larger particles $(0.01$ to $0.1 \mathrm{~mm})$, and an increase by threefold of particles 0.1 to $1.0 \mathrm{~mm}$ in the center of the belt compared to near-Earth regions. Then Pioneer 11 made its closest encounter with Jupiter on December 2, 1974, at $43,000 \mathrm{~km}$ above the cloud tops and proceeded on a trajectory toward Saturn. A brief summary of the experimental results obtained by both spacecraft from the encounter with Jupiter is listed in Table 3. On September 2, 1979, the Pioneer 11 spacecraft made its closest approach to Saturn at $20,930 \mathrm{~km}$ above the cloud tops $3,4 /$. A synopsis of these results is given in Table 4. After its Saturn encounter, the Pioneer 11 spacecraft is proceeding out to deep space in the same direction as the Sun's motion in our galaxy.

The next phase of the Pioneer 10 and 11 missions involves the longest period of their lifetime and is devoted to sudying the heliosphere /5/. This region is dominated by the plasma envelope of the Sun and is thought to be on the order of $100 \mathrm{AU}$ in distance before this solar plasma interacts with the interstellar plasma. More than one complete solar cycle has elapsed during the 17 years that the two Pioneers have been measuring the solar wind ions and magnetic field and the cosmic ray energetic particles in this extremely large region of space. Interpretation of these measurements depends upon baseline measurements in the region near the Sun obtained by three other spacecraft: 
TABLE 3 Jupiter Encounter

1. Measurements of the magnetic field indicated 4.28 gauss at the equatorial 1 bar level, and the dipole tilted $11^{\circ}$ with respect to the spin axis.

2. Plasma and magnetic field measurements show that a strong ring current distorts the magnetosphere. A bow shock was detected at $100 \mathrm{R}$.

3. The electron flux trapped in the Jovian magnetic field was $10^{4}$ times greater than the Earch's and peaked at $3 R_{\mathrm{J}}$. The trapped protons were $10^{3}$ times more intense than the Earth's and peaked in two regions; $3.4 \mathrm{R}_{\mathrm{J}}$ and $1.9 \mathrm{R}_{\mathrm{J}}$.

4. The energetic particle experiments discovered that Jupiter is the dominant source of low-energy relativistic electrons in the heliosphere and the intensity and spectrum varies with the 10-hr rotation period of Jupiter.

5. Infrared radiometer measurements showed that Jupiter emits 1.7 times more heat than it receives from the Sun. The helium to hydrogen ratio was measured to be $0.14 \pm 0.08$.

6. Close-up observations of Jupiter's atmosphere showed that the cloud tops at the poles are lower than those at the equator and that atmospheric convection cells are smallest in the polar regions.

7. Energetic particle absorption measurements indicated the existence of a ring of particulate matter around Jupiter.

8. Spacecraft Doppler tracking significantly improved our knowledge of the gravity field of Jupiter and determined the masses of the Galilean satellites with a $20 \%$ improvement in accuracy.

TABLE4 Sanum Encounter

1. The magnetic field was measured to be 0.22 gauss at the equatorial 1 bar level with the dipole axis tilted less than $1^{\circ}$ to the spin axis.

2. Saturn's magnetosphere contained a plasma that was rigidly corotating with the planet in regions less than 10Rs. Beyond this region the plasma was corotating at speeds less than that of the planet.

3. Energetic particle experiments discovered that the trapped radiation belts had comparable intensity to those of Earth, but extended over much larger regions. The maximum intensity of electrons and protons were found to be between 4Rs and the outer edge of the rings at 2.292Rs. The proton flux peaked at 2.67Rs.

4. The infrared radiometer measurements showed that Satum emits $2.8 \pm 0.9$ times more heat than it receives from the Sun.

5. A new ring designated $\mathrm{F}$ was discovered by imaging and energetic particle absorption measurements.

6. A new inner Saturnian satellite named Epimethens was detecred by imaging and energetic particle absorption.

7. The close-up imaging of Saturn showed that the clouds are thicker than those of Jupiter and have much less contrast. The cloud belis are also narrower and more numerous than those of Jupiter.

8. Accurate Doppler tracking of the spacecraft permitted the masses of Saturn, Lapetus, Rhea, and Titan to be determined more precisely. 
the Interplanetary Monitoring Platform (IMP-8), the International Cometary Explorer (ICE), and the Pioneer Venus Orbiter (PVO). The main scientific objectives of these spacecraft are to examine the following: (1) solar wind structure and dynamics, (2) interaction of the solar wind with the interstellar medium, and (3) acceleration, transport, and modulation of energetic particles. A brief list of the results obtained during the last 17 years in the heliosphere is given in Table 5. These results have significantly changed the models of the heliosphere developed in the early 1960s and have produced a much better understanding of the origin and transport of cosmic radiation.

Future objectives of the Pioneer 10 and 11 missions are listed in Table 6. Accomplishment of these objectives is strongly dependent on the RTG spacecraft power supplies and the continued allocation of the Deep Space Network of 70-m antenna stations required to command and track the two spacecraft. The continued operation of the Pioneer 10 and 11 spacecraft in conjunction with Voyager 1 and 2, and later, Galileo and Ulysses, provides a unique capability to study the heliosphere during this era.

\section{TABLE 5 Heliospheric Measurements}

1. Measurements of the speed, number density, and temperature of the solar wind out to $47 \mathrm{AU}$ show that the mean velocity is constant $-430 \mathrm{~km} / \mathrm{sec}$, the mean density falls off as $R^{-2}$, and that the temperature gradient is complicated but always subadiabatic.

2. The average structure of the heliosphere substantiates the Parker model with a magnetic field drawn out to form Archimedean spirals in the ecliptic plane and dipole-like asymmetry in the polar directions. However, the azimuthal magnetic component shows a $20 \%$ departure from the model at $20 \mathrm{AU}$.

3. The magnetic field strength observed in the outer heliospheres is a minimum at solar minimum and the inclination of the current sheet is near equatorial at solar minimum.

4. The solar wind stream morphology evolves from corotating interaction regions at 5 to $10 \mathrm{AU}$ into lower velocity stream with broad high-density regions beyond 20 AU.

\section{Strong repeated flaring regions on the Sun produce very strong shocks at large distances (R > 27 AU).}

6. Energetic particle measurements of the intensity and radial gradient of galactic cosmic rays indicate a "modulation boundary" at 40 to $80 \mathrm{AU}$.

7. An "anomalous component" of the cosmic ray spectrum between 5 to $50 \mathrm{MeV} /$ nucleon was discovered for $\mathrm{He}, \mathrm{C}, \mathrm{N}, \mathrm{O}$, and $\mathrm{Ne}$. The source is thought to be interstellar neutrals which penetrate the inner heliospheres, become ionized, and are transported to the outer heliosphere where the ionized particles are accelerated by a factor of $10^{4}$.

8. The energetic particle radial gradient changes only slightly over the solar cycle while the intensity varies by a significant factor. This indicates that the distance of the modulation boundary from the Sun depends upon the phase of the solar cycle.

9. Energetic particle modulation in the outer heliosphere takes place in a series of step decreases propagating outward at $-50 \mathrm{~km} / \mathrm{sec}$ and are produced by merged interaction regions beyond $8 \mathrm{AU}$.

10. UV photometer measurements on Pioneer 10 of the hydrogen and helium density values in the distant heliosphere show an abrupt drop in the flux of Lyman alpha photons. This has been interpreted as being caused by enhanced charge exchange between solar wind protons and the interstellar hydrogen. 
1. Utilize spacecraft power sharing and enhanced capabilities of the deep space network to keep the Pioneer experiments working until the year 2000.

2. Measure the radial evolution of the solar wind.

3. Determine the latitudinal variation in the plasma and magnetic field.

4. Measure the interaction of the solar wind with the incoming interstellar neutral particles.

5. Determine the existence and location of a terminal shock and the heliopause.

6. Determine the location and acceleration mechanism for the anomalous component of cosmic rays.

7. Measure the temporal, radial, and latitudinal variations in the strength of cosmic ray modulation.

8. Use accurate Doppler tracking to search for gravitational radiation and differential gravitational forces from a possible trans-Neptunian planet.

\section{REFERENCES}

1. G. D. Mead, Pioncer 10 Mission: Jupiter Encounter Special Issue, L Geophys, Res, 79 \#A25, 3487-3886 (1974).

2. T. G. Northrop, A.G. Opp, and J. H. Wolfe, Pioneer 11 Saturn Encounter Special Issue, L Geophys. Res, 85 \#A11. $5651-5958$ (1980).

3. R. D. Fimmel, J. Van Allen, and E. Burgess, Bieneer First te Jupiter. Saturm. and Beyond. NASA SP-446, 1980.

4. D. Morrison, Yoyazes to Samum. NASA SP-451, 1982.

5. P. Dyal and R. O. Fimmel, Bioneer 10 and 11 Heliospheric Mission Ames Research Center, Moffen Field, CA 94035, (1982). 


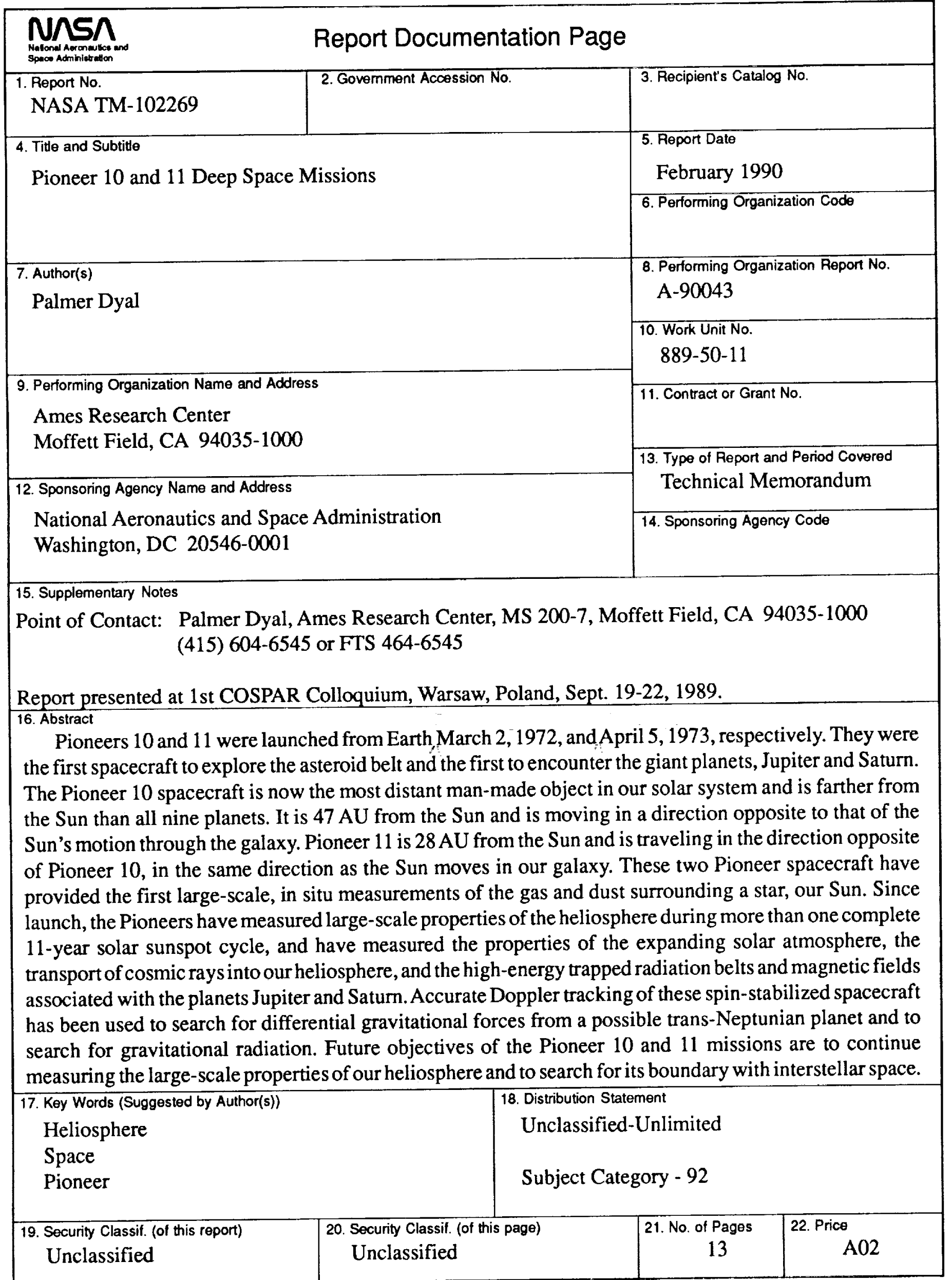


